\title{
Wiener-Typed Nonlinear Systems' Output Feedback Control Algorithm Based on Dual-Mode Method
}

\author{
Hai-Tao Zhang, Yong-Ji Wang, Member,IEEE and Hua O. Wang, Senior Member, IEEE
}

\begin{abstract}
Wiener-typed nonlinear systems with hard input constraints are ubiquitous in industrial processes. However, owing to their complex structures, there are very few achievements on their control algorithm. Aimed at this problem, an improved dual-mode control algorithm is put forward. Firstly, the detailed procedure of this algorithm is proposed. Then, its feasibility, stability and convergence are analyzed by using the invariant set theory combined with LMI(linear matrix inequalities) technique ${ }^{[8]}$. In contrast to traditional algorithms, this one has the capabilities of maximizing the size of the closed-loop stable region and decreasing the online computational burden. Finally, the proposed algorithm is performed by simulations with promising results.
\end{abstract}

Key words - Dual-Mode control algorithm, Wiener-typed nonlinear systems, Zeroin algorithm, invariant set, LMI

\section{INTRODUCTION}

$\mathrm{D}^{2}$ uring a lot of real industrial processes such as distillation $^{[9]}$, $\mathrm{pH}$ neutralization control ${ }^{[2,5]}$, heat-exchanger ${ }^{[16]}$, chemical reaction ${ }^{[14]}$, and biological visual process $^{[16,17]}$, there widely exists a type of nonlinear systems which can be described by Wiener mode. The background of this model can be even extended to the areas of communication $^{[11]}$, signal processing ${ }^{[11]}$, psychology ${ }^{[17]}$ and sociology ${ }^{[17]}$. It consists of a linear dynamic element followed by a memoryless nonlinear element while Hammerstein model contains the same elements in the reverse order ${ }^{[9]}$. Wiener-typed systems correspond to processes with linear dynamics associated with general nonlinear operators. In recent years, the control of Wiener-typed systems has become one of the most urgent and difficult tasks in nonlinear control field $^{[2,5,9,11,16,17]}$.

Due to the particular structure of Wiener model, the identification and control algorithms of this system are much more complicated than the counterparts of Hammerstein-typed system. Suny and Lee ${ }^{[18]}$ worked over the method approaching nonlinear element with polynomials based on the suggestion that the output of linear block be feasible. However, in practical industrial control, this

Haitao Zhang is with the department of Control Science and Engineering, Huazhong Univ. of Science and Technology, Wuhan, Hubei Province, 430074, P.R.C. (zht@ mail.hust.edu.cn).

Yongji Wang is with the department of Control Science and Engineering, Huazhong Univ. of Science and Technology, Wuhan, Hubei Province, 430074, P.R.C. (corresponding author to provide phone: 86-27-87540014; wangyjch@mail.hust.edu.cn).

Hua O. Wang is with the department of Aerospace and Mechanical Engineering, Boston Univ., Boston, MA02215, USA(wangh@bu.edu). assumption can not be always satisfied. In $1997^{[9]}$, Kalafatis and Wang proposed a method identifying the two parts of Wiener model at the same time, but an assumption must be satisfied that the inverse of the nonlinear element can be approximated by $P$ order polynomials with satisfactory precision, which greatly limited its applications. In the same year $^{[14]}$, Yamanaka et al developed a new kind of dynamic neural network which is composed by a Laguerre function filter and a memoryless nonlinear block. Based on this model they presented a model reference adaptive control scheme for Wiener-typed nonlinear systems. Stability analysis was also given, but these conditions were too rigid and the initial weights were difficult to optimize. In $2001^{[7]}$, Duwaish et al developed an approach to control Wiener models using MPC combined with genetic algorithm, but the computational burden was so heavy that its real-time performances were not satisfactory.

It can be concluded that most of the existing control algorithms for Wiener-typed systems have somewhat limitations. Moreover, there are very few achievements on dealing with hard input constraints so far. However, this type of constraints exists universally in industrial processes because it is the physical restriction of the executers, which can not be violated ${ }^{1,2,5,7,9,5,14-18]}$. Therefore, it is a fairly valuable work to find an effective solution for this problem. In $2000^{[12]}$, Yang et al proposed an approach based on dual-mode technique to control Hammerstein-typed systems with hard input constraints, and gained excellent performances. Nevertheless, owing to the complexity of Wiener-typed systems, they have not taken into consideration of the hard input constraints.

In this paper, based on dual-mode technique ${ }^{[8]}$, we propose an output feedback control method. The main two contributions of this paper can be summarized as follows, 1) For system with hard input constraints, Dual-Mode control technique $^{[8]}$ is imported to enlarge the stable region. In detail, this algorithm has two modes, Mode 1) when the initial state is outside the initial invariant set, the system state is extend by an auxiliary vector which can drive the state into the initial stable invariant set in finite steps; Mode 2) Once the state enters this stable set, the auxiliary vector decreased to zero automatically, and the standard stable output feedback control law is applied to drive the system state to the origin asymptotically.

2)The closed-loop stability is proven based on invariant set theory ${ }^{[6]}$ combined with LMI (linear matrix inequalities) method.

This paper is organized as follows. In section II, the 
problem description is given. The control algorithms are presented in sections III and IV. Also in these two sections, the stability and convergence analyses are also presented. In section $\mathrm{V}$, simulations are performed with promising results. Finally, conclusion remarks are given in section VI.

\section{PRoblem DESCRIPTION}

\section{A. Problem description in Z-domain}

A Wiener-typed system consists of a linear dynamic element followed by a memoryless nonlinear element. The structure of this model in Z-domain is shown in figure 1. The difference equation of this system is described as

$$
\left\{\begin{array}{l}
a\left(z^{-1}\right) \eta(k)=z^{-d} b\left(z^{-1}\right) u(k) \\
y(k)=f[\eta(k)]
\end{array}\right.
$$

with $\left\{\begin{array}{c}a\left(z^{-1}\right)=1+a_{1} z^{-1}+\cdots+a_{n} z^{-n} \\ b\left(z^{-1}\right)=b_{0}+b_{1} z^{-1}+\cdots+b_{m} z^{-m}\end{array}\right.$

$d$ is system's time delay, $f(\cdot)$ is a memoryless nonlinear function, $u(k), y(k)$ and $\eta(k)$ are the input, output and output of dynamic linear element respectively.

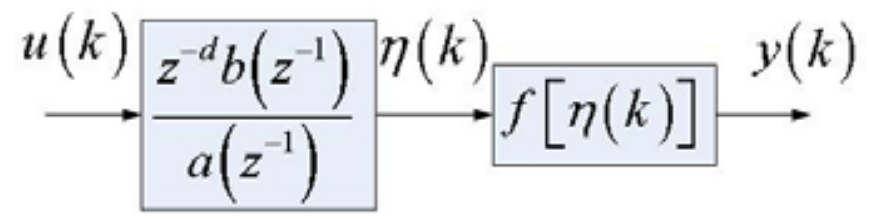

Fig.1 Structure of Wiener-typed system

\section{B. Problem description in state space}

Assume the linear element of our Wiener-typed plant can be described by

$$
\begin{gathered}
x(k+1)=A x(k)+B u(k) \\
\eta(k)=C x(k)
\end{gathered}
$$

There are two kinds of control problems

P1(Regulator Problem) to make the origin asymptotically

stable, then one can design $u(k)=K x(k)$, then

$$
x(k+1)=\Psi x(k)
$$

with

$$
\Psi=A+B K \text {. }
$$

P2(Tracking Problem) to track a set point $r(k)$, then one can design

$$
u(k)=K x(k)+r(k) \theta
$$

with

$$
1 / \theta=\lim _{z \rightarrow 1}\left(C(z I-\Psi)^{-1} B\right) .
$$

Reference [3] has proven that, if each eigenvalue of $\Psi$ is inside the unit circle of complex plane in Z-Domain, then the control law (5) can drive system output to the set point $r(k)$, and make system globally uniformly asymptotically stable. Remark 1: These two control problems are equivalent, the reason is given as follows. If $(I-\Psi)$ is nonsingular, then one can make the coordinate transformation $x(k)=x(k)-\alpha$, with $\alpha=(I-\Psi)^{-1} B r \theta$, and still obtain (4).Else, i.e. $(I-\Psi)$ is singular, one can still find suitable $\alpha$ to obtain (4). Thus, the two control problems are equivalent. Therefore, without loss of generality, we will only discuss the problem $\boldsymbol{P 1}$ ) in the following text.

Then, the problems this paper addresses are motivated naturally:

1) If the prior knowledge of the Wiener-typed system's memoryless nonlinear block, is partially known at least, how to guarantee the closed-loop stability of the Wiener-typed system subjected to hard input constraints

$u_{\text {min }} \leq u \leq u_{\text {max }}$ ?

2) Furthermore, how to maximize the size of abovementioned closed-loop stable region?

\section{OUTPUT FEEDBACK CONTROL ALGORITHM FOR SYSTEMS WITHOUT HARD INPUT CONSTRIANTS}

\section{A. Conditions of invariant sets}

Firstly, assume the state observer be given by

$\hat{x}(k+1)=A \hat{x}(k)+B u(k)+L(\tilde{\eta}(k)-C \hat{x}(k))$

where $\hat{x}(k)$ is the estimation state, and $\widetilde{\eta}(k)=f_{\text {Zeroin }}^{-1}(y(k))$, in which $f_{\text {Zeroin }}^{-1}$ refers to the inverse of $f$ calculated by Zeroin algorithm ${ }^{[4]}$.

The estimation state error is defined as

$$
e(k) \triangleq x(k)-\hat{x}(k)
$$

Then, one can define the invariant ellipsoid sets of estimation state and estimation state errors as follows

$$
\begin{gathered}
S \triangleq\left\{\hat{x} \mid \hat{x}^{T} P \hat{x} \leq 1\right\} \\
S_{e} \triangleq\left\{e \mid e^{T} P_{e} e \leq \bar{e}^{2}\right\}, 0 \leq \bar{e}^{2} \leq 1
\end{gathered}
$$

where $P_{e}$ and $P$ are positive definite symmetric matrices.

When $\hat{x}(k) \in S, e(k) \in S_{e}$, in order to make these ellipsoid sets invariant, it is required that

$$
\begin{gathered}
\hat{x}^{T}(k+1) P \hat{x}(k+1) \leq 1 \\
e^{T}(k+1) P_{e} e(k+1) \leq \bar{e}^{2}
\end{gathered}
$$

Provided the control law $u(k)=K \hat{x}(k)$, then (7) can be rewritten as

$$
\hat{x}(k+1)=\Psi \hat{x}(k)+L(\tilde{\eta}(k)-C \hat{x}(k))
$$

Assume that $\tilde{\eta}(k)-\eta(k)=\delta[\eta(k)] C e(k)$

then substituting (14) to (13) yields

$$
\hat{x}(k+1)=\Psi \hat{x}(k)+(1+\delta[\eta(k)]) L C e(k)
$$

and substituting (15) to (11) yields 


$$
\begin{gathered}
(\Psi \hat{x}(k)+L C(1+\delta[\eta(k)]) e(k))^{T} P . \\
(\Psi \hat{x}(k)+L C(1+\delta[\eta(k)]) e(k)) \leq 1
\end{gathered}
$$

Lemma $1^{[11]}$ : For $\forall \mu>1(\mu \in R), \tau=1+1 /(\mu-1)$, and $a, b$ are matrices with the same size, then

$$
(a+b)^{T} P(a+b) \leq \mu a^{T} P a+\tau b^{T} P b
$$

Theorem 1: The linear time-invariant block of the Wiener-typed nonlinear system (1) is shown as (2), (3), and the state space description of the observer is given as (7), where the feedback gain $K$ and the observer gain $L$ are stable. Then, if the following three assumptions $A 1$ ) and $A 2$ ) are fulfilled, it can be conclude that $S$ and $S_{e}$ are invariant sets of $x(k)$ and $e(k)$ in the sense of (9) and (10), respectively.

A1) (14) is satisfied, in addition, there exists $\sigma>0$ $(\sigma \in R)$ such that $\quad|\delta[\eta(k)]| \leq \sigma$

A2) there exist $\mu>1, \mu_{1}>1,\left(\mu, \mu_{1} \in R\right)$ such that

$$
\begin{gathered}
\mu_{1} \Psi^{T} P \Psi \leq\left(1-\bar{e}^{2}\right) P \\
\tau_{1}(1+\sigma)^{2} C^{T} L^{T} P L C \leq P_{e} \\
\mu \Psi^{T} P_{e} \Psi+\tau \sigma^{2} C^{T} L^{T} P_{e} L C \leq P_{e}
\end{gathered}
$$

with $\tau=1+1 /(\mu-1), \tau_{1}=1+1 /\left(\mu_{1}-1\right)$.

\section{Proof:}

For convenience, we use $\delta(\cdot)$ to donete $\delta[\eta(k)]$.

From (8), we can get

$$
e(k+1)=x(k+1)-\hat{x}(k+1)
$$

Then substituting (4) and (13) to the above equation yields

$$
e(k+1)=[\Psi-L C(1+\delta(\cdot))] e(k)
$$

From Lemma1, and take into consideration of (9), (18), (19) and (20), we have that for $\forall \mu_{1}>1, \tau_{1}=1+1 /\left(\mu_{1}-1\right)$

$\hat{x}^{T}(k+1) P \hat{x}(k+1)=$

$[\Psi \hat{x}(k)+L C(1+\delta(\cdot)) e(k)]^{T} P[\Psi \hat{x}(k)+L C(1+\delta(\cdot)) e(k)] \leq$

$\mu_{1} \hat{x}^{T}(k) \Psi^{T} P \Psi \hat{x}(k)+\tau_{1}[1+\delta(\cdot)]^{2} e^{T}(k) C^{T} L^{T} P L C e(k) \leq$ $\left(1-\bar{e}^{2}\right) \hat{x}^{T} P \hat{x}+\tau_{1}(1+\sigma)^{2} e^{T}(k) C^{T} L^{T} P L C e(k) \leq$ $1-\bar{e}^{2}+\bar{e}^{2}=1$

By using Lemma1 combined with (24), we have for

$\forall \mu>1, \tau=1+1 /(\mu-1)$

$$
[(\Psi-L C \delta(\cdot)) e(k)]^{T} P_{e}[(\Psi-L C \delta(\cdot)) e(k)]
$$

$\leq \mu e^{T}(k) \Psi^{T} P_{e} \Psi e(k)+\tau \delta(\cdot)^{2} e(k)^{T} C^{T} L^{T} P_{e} L C e(k)$

$\leq e^{T}(k)\left[\mu \Psi^{T} P_{e} \Psi+\tau \sigma^{2} C^{T} L^{T} P_{e} L C\right] e(k)$

Therefore, substituting (21) to (26) yields

$$
e^{T}(k+1) P_{e} e(k+1) \leq \bar{e}^{2}
$$

so $S_{e}$ is an ellipsoid invariant set of $e(k)$.

Remark 2: assumption $\boldsymbol{A 1}$ ) is reasonable, because the linear element's output estimation error can be rewritten as the sum of two parts, i.e. error part I and error part II as follows

$$
C e(k)=\eta-C \hat{x}=\underbrace{\left[\eta-f_{\text {Zeroin }}^{-1}(f(\eta))\right]}_{\text {error part I }}+\underbrace{\left[f_{\text {Zeroin }}^{-1}(f(\eta))-C \hat{x}\right]}_{\text {eror part II }} \text { (28) }
$$

Hence, it is reasonable to assume that error part I be proportional to $\mathrm{Ce}(k)$ in the $k^{\text {th }}$ sample time, and the proposition $\delta(\cdot)$ is determined by $\eta(k)$. Moreover, it is also reasonable to assume that $\delta(\cdot)$ is bounded, since if enough prior knowledge about the memoryless nonlinear block is available, Zeroin algorithm can guarantee the accuracy of $f_{\text {Zeroin }}{ }^{-1}(.){ }^{[4]}$. Then the error $C e(k)$ is mainly caused by the state estimator $L$.

\section{OUTPUT FEEDBACK CONTROL ALGORITHM FOR SYSTEMS WITH HARD INPUT CONSTRIANTS}

\section{A. Dual Mode technique and conditions of invariant sets}

For a Wiener-typed system subjected to $u_{\text {min }} \leq u \leq u_{\text {max }}$, we extend $\hat{x}(k)$ by auxillary vector $D(k)=\left[d_{1}(k), \cdots, d_{n_{d}}(k)\right]^{T}$. Thus, the extended state vector is $\hat{z}(k)=\left[\hat{x}^{T}(k), D^{T}(k)\right]^{T}$. Then one can design the stable output feedback control law as

$$
u(k)=K \hat{x}(k)+E D(k)
$$

with $E=[1,0, \cdots, 0]_{1 \times n_{d}}, D(k)=\left[d_{1}(k), \cdots, d_{n_{d}}(k)\right]^{T}$, then substituting (14) and (29) to (7) yields

$$
\hat{x}(k+1)=\Psi \hat{x}(k)+B E D(k)+L C[1+\delta(\cdot)] e(k)
$$

where $D(k)$ is gained by solving the following QP question

$$
\begin{aligned}
& \min _{D(k)} J=D^{T}(k) D(k) \\
& \text { s.t. }\left\{\begin{array}{l}
\hat{z}^{T}(k) \bar{P} \hat{z}(k)<1 \\
u_{\min } \leq u(k) \leq u_{\max }
\end{array}\right.
\end{aligned}
$$

Take the extended state $\hat{z}(k)$ into consideration, we obtain 


$$
\hat{z}(k+1)=\Xi \hat{z}(k)+\left[\begin{array}{c}
L C[1+\delta(\cdot)] \\
0
\end{array}\right] e(k)
$$

with

$$
\begin{gathered}
\Xi=\left[\begin{array}{cc}
A+B K & B E \\
0 & M
\end{array}\right], M=\left[\begin{array}{cc}
\vartheta & I \\
0 & \vartheta^{T}
\end{array}\right]_{n_{d} \times n_{d}}, \\
\vartheta=[0, \cdots, 0]_{\left(n_{d}-1\right) \times 1}^{T}, I=\operatorname{diag} \underbrace{(1, \cdots, 1)}_{n_{d}-1}
\end{gathered}
$$

Define ellipsoid invariant set of the estimation extended state

$$
\bar{S} \triangleq\left\{\hat{z} \mid \hat{z}^{T} \bar{P} \hat{z} \leq 1\right\}
$$

where the $\bar{P}$ is a positive define symmetric matrix.

Theorem 2: The linear block of the Wiener-typed system (1) is shown as (2) and (3), and the state space expression of the observer is given by (7), where the feedback vector gain $K$ and the observer gain $L$ are stable. In addition, the assumption $\boldsymbol{A 1}$ ) is satisfied for the Wiener-typed system (1) subjected to the hard input constraints $u_{\text {min }} \leq u \leq u_{\text {max }}$. Then, if the following assumption $\boldsymbol{A 3}$ ) is satisfied, $\bar{S}$ and $S_{e}$ are invariant sets in the sense of (33) and (10), respectively.

A3) There exist $\mu>1, \tilde{\mu}>1,(\mu, \tilde{\mu} \in R)$ such that

$$
\begin{gathered}
\tilde{\mu}^{T} \Xi^{T} \bar{P} \leq\left(1-\bar{e}^{2}\right) \bar{P} \\
\tilde{\tau}\left(1+\sigma^{2}\right) C^{T} L^{T} E_{x}^{T} \bar{P} E_{x} L C \leq P_{e} \\
\mu \Psi^{T} P_{e} \Psi+\tau \sigma^{2} C^{T} L^{T} P_{e} L C \leq P_{e}
\end{gathered}
$$

with $\tau=1+1 /(\mu-1), \tilde{\tau}=1+1 /(\tilde{\mu}-1), E_{x}$ is a transform factor such that $\hat{x}=E_{x}^{T} \hat{z}$.

Proof: For $\forall \hat{z}(k) \in \bar{S}$, we conclude from (33) and (35) that $\tilde{\mu} \hat{z}(k) \Xi^{T} \bar{P} \Xi \hat{z}(k) \leq\left(1-\bar{e}^{2}\right) \hat{z}^{T}(k) \bar{P} \hat{z}(k) \leq 1-\bar{e}^{2}$

For $\forall e(k) \in S_{e}$, from (11), (18) and (34), we have

$$
\begin{aligned}
& \tilde{\tau} e^{T}(k)[1+\delta(\cdot)]^{2} C^{T} L^{T} E_{x}^{T} \bar{P} E_{x} L C e(k) \leq \tilde{\tau} e^{T}(k)(1+\sigma)^{2} . \\
& C^{T} L^{T} E_{x}^{T} \bar{P} E_{x} L C e(k) \leq e^{T}(k) P_{e} e(k) \leq \bar{e}^{2}
\end{aligned}
$$

Moreover, applying lemma 1, we have

$$
\begin{aligned}
& \hat{z}^{T}(k+1) \bar{P} \hat{z}(k+1) \leq \tilde{\mu} \hat{z}^{T}(k) \Xi^{T} \bar{P} \Xi z(k)+ \\
& \tilde{\tau} e(k)^{T}\left(1+\delta(\cdot)^{2}\right) C^{T} L^{T} E_{x}^{T} \bar{P} E_{x} L C e(k) \quad(\forall \hat{z}(k) \in \bar{S})
\end{aligned}
$$

Substituting (37) and (38) to (39) yields

$$
\hat{z}^{T}(k+1) \bar{P} \hat{z}(k+1) \leq 1
$$

Hence, $\bar{S}$ is an invariant set of extended state $\hat{z}(k)$. On the other hand, like the proof of theorem 1, we can gain from (27) and (36) that $S_{e}$ is an invariant set.

Remark $3^{[11]}$ : If the input constraints are symmetric, i.e. $|u(k)| \leq \bar{u}$, then
$|u(k)|=|K \hat{x}(k)+E D(k)|=|\bar{K} \hat{z}(k)|=\left|\bar{K} \bar{P}^{-1 / 2} \bar{P}^{1 / 2} \hat{z}(k)\right| \leq$
$\left\|\bar{K} \bar{P}^{-1 / 2}\right\| \cdot\left\|\bar{P}^{1 / 2} \hat{z}(k)\right\| \leq \bar{u}$

with $\bar{K}=[K, E]$

From (33), we have $\quad\left\|\bar{P}^{1 / 2} \hat{z}(k)\right\| \leq 1$

Then, substituting (41) to (40) yields

$$
\left\|\bar{K} \cdot \bar{P}^{-1 / 2}\right\| \leq \bar{u} \Leftrightarrow\left[\begin{array}{cc}
\bar{P} & \bar{K}^{T} \\
\bar{K} & \bar{u}^{2}
\end{array}\right] \geq 0
$$

thus, these constraints can be solved offline, which reduces the computational load remarkably. Otherwise, i.e.

$u_{\text {min }} \leq u \leq u_{\text {max }}\left(u_{\text {min }} \neq-u_{\text {max }}\right)$, it is required to solve

these constraints online.

\section{B. Stability analysis}

Theorem 3: If the following three assumptions are fulfilled A4) in initial time, there exist $\bar{P}$ and $P_{e}$ such that (33) and (12) are fulfilled respectively;

A5) each eigenvalue of $\Phi=A-L C[1+\delta(\cdot)]$ is inside the unit circle of the complex plane in Z-domain, where $\delta(\cdot)<\sigma(\exists \sigma>0)$ when $u_{\text {min }} \leq u \leq u_{\text {max }}$

A6) each eigenvalue of $\Psi$ is inside the unit circle.

Then our algorithm is always feasible and the system is closed-loop asymptotically stable. Moreover, the control law (29) will converge to the standard stable output feedback control law $u(k)=K \hat{x}(k)$.

\section{Proof:}

From A4)-A6), if in the $k^{\text {th }}$ sample time, state $z(k) \in \bar{S}$. The definition of $\bar{S}$, i.e. (33), implies that there must exist $D(k+1)$ such that $z(k+1) \in \bar{S}$. From (31), one can obtain $\bar{D}(k+1)=M D(k)$, and the $i^{\text {th }}$ element of $\bar{D}(k+1)$ equals to the $(i+1){ }^{\text {th }}$ element of $D(k)$, moreover, the $n_{d}{ }^{\text {th }}$ element of $M D(k)$ is 0 . Hence, in the $k^{\text {th }}$ sample time, if there exists a feasible $D(k)$, then in the $(k+1)^{\text {th }}$ sample time there must exist a feasible $\bar{D}(k+1)$ such that $\bar{J}(k+1)=\bar{D}^{T}(k+1) \bar{D}(k+1) \leq \bar{J}(k)$, where $\bar{J}(k+1)=\bar{J}(k)$ only if $D(k)=[0 \cdots 0]_{n_{d} \times 1}^{T}$. In the $(k+1)^{\text {th }}$ sample time, it is evident that $\bar{D}(k+1)$ is not always the optimized solution, and the optimized solution $D^{*}(k+1)$ gained form $J(k+1)$ will make

$$
J(k+1) \leq \bar{J}(k+1) \leq J(k) .
$$


In this way, the performance index $J(k)$ decreases monotonously, and $D(k)$ will converge to 0 with the increase of sample time. In other words, the constrained control law $u=K \hat{x}+E D$ will converge to unconstrained stable control law $u=K \hat{x}$. Consider the assumptions $\boldsymbol{A 5}$ ) and $\boldsymbol{A 5}$ ) combined with Separation Principle ${ }^{[3]}$, we can conclude that the closed-loop asymptotical stability of this system is guaranteed.

\section{CAse Study}

Plant:

$$
\begin{gathered}
x(k+1)=A x(k)+B u(k) \\
\eta(k)=C x(k) \\
y(k)=\eta^{4}(k) \sin [\eta(k)]-\eta^{5}(k) \\
\text { s.t. }-1.5 \leq u(k) \leq 3
\end{gathered}
$$

with $A=\left[\begin{array}{cc}2.3 & -1.2 \\ 1 & 0\end{array}\right], B=\left[\begin{array}{l}1 \\ 0\end{array}\right], C=\left[\begin{array}{ll}1 & 0\end{array}\right]$.

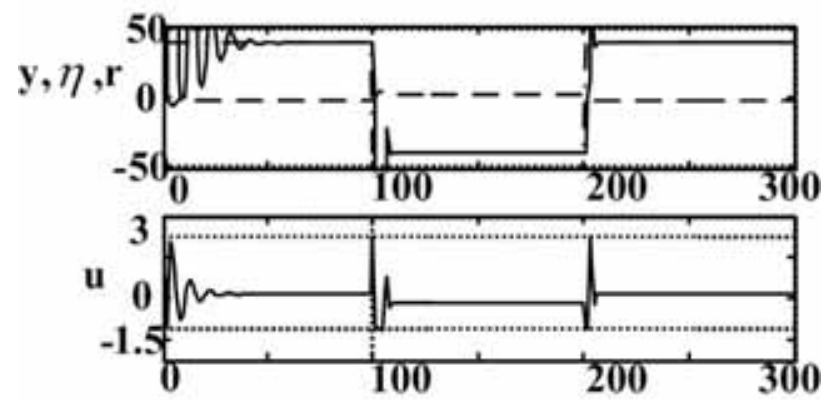

Fig.2 Control performance tracking step signals

Apply the DLQR function of Matlab to design the state feedback controller: $K=\left[\begin{array}{ll}-2.4179 & 1.1495\end{array}\right]$; meanwhile, apply the DLQE function of Matlab6 to design the state observer: $L=\left[\begin{array}{ll}1.0556 & 0.3704\end{array}\right]^{T}$. Figure 2 shows the control performance of tracking $\{40,-40\}$ double-step signals.

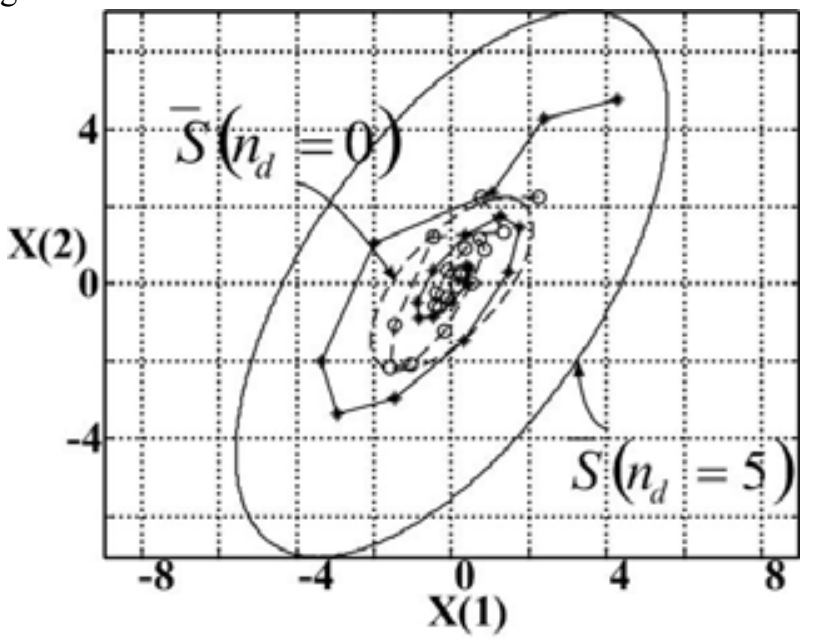

Fig.3 Tracks and ellipsoid invariant sets of the state and estimation state
Parameter settings: $n_{d}=5, \bar{e}=0.4, x(0)=\left[\begin{array}{ll}2.0 & 2.5\end{array}\right]^{T}$ $\mu=1.1, \tilde{\mu}=1.5, \sigma=0.1$. In figure 2 , the upper subfigure denotes $r$ (dashed-dot line: set points), $y$ (solid line: output), and $\eta$ (dashed line: linear block's output), respectively; the middle subfigure denote $u$ (control variable); and the lower subfigures denote $D(1)$ (the first element of $D$ ). These results validate the feasibility of our proposed dual-mode control algorithm. However, because the constraints are too strict, initially the overshootings are something big. But they can be alleviated by enlarging the size of the input space or selecting more suitable parameters.

Figure 3 shows that the tracks and ellipsoid invariant sets of $x$ and $\hat{x}$, where the solid ellipsoid refers to $\bar{S}$ with $n_{d}=5$, the dashed ellipsoid is $\bar{S}$ with $n_{d}=0$, the dashed lines and circular points are the track of $\hat{x}$, and the solid lines and starlike points denote the track of $x$. For convenience, only the first 20 states and estimation states are drawn. The tracks of $x$ and $\hat{x}$ begin inside the dashed ellipsoid, then enter the solid one only after 6 epochs, and 2 epochs respectively. From then on, the standard output control law can drive the state to the origin. In this way, the superiority of our algorithm is verified.

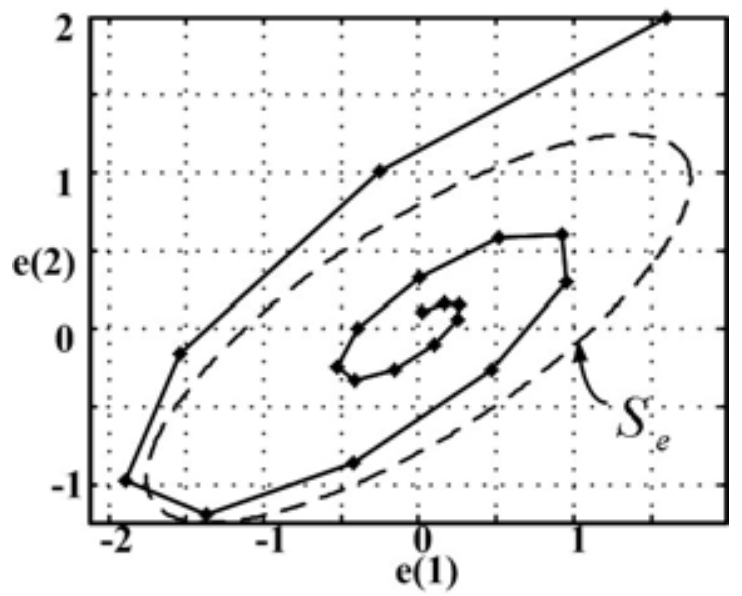

Fig.4 Tracks and ellipsoid invariant sets of the estimation state error

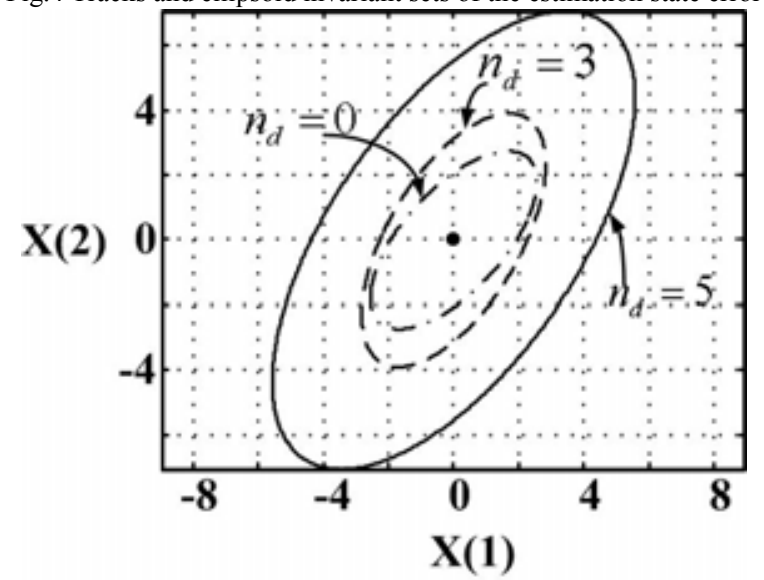

Fig.5. Ellipsoid invariant sets of the state for different $n_{d}$ 
Figure 4 shows the track and ellipsoid invariant set of the estimation error $e$, where the purple ellipsoid denotes the $S_{e}$ (see (10)). The solid lines and starlike points denote the track of $e$. Like figure 3, only the first 20 estimation errors are shown. Although the track of $e(k)$ starts outside $S_{e}$, only after 3 epochs, this track moves into $S_{e}$ and then asymptotically converges to the origin, which also shows the high convergent speed of the algorithm. Therefore,

Theorems $1 \mathbf{- 3}$ can also be validated by Figures 3 and 4 . Still worth mentioning is that, as is shown in figure 5, $\bar{S}$ will grow larger along with the increase of $n_{d}$. However, in order to gain satisfactory real-time performances, one can not select too big $n_{d}$. If a tradeoff $n_{d}$ between the computational load and the size of closed-loop region is selected, the control performance of this algorithm will be further improved.

Remark 4: In order to show the superiority of this proposed algorithm clearly, state $x$ and estimation state $\hat{x}$ in figures 3 and 5 have been transformed by $x(k)=x(k)-\alpha$, and $\hat{x}(k)=\hat{x}(k)-\alpha$.This coordinate transform is shown in Remark 1.

\section{CONCLUSION}

For time-invariant Wiener-typed nonlinear systems subjected to hard input constraints, a control algorithm based on dual-mode technique is proposed in this paper. Firstly, Zeroin algorithm is applied to solve the nonlinear equation. Then, the system state is extended and then invariant set theorem combined with LMI is used to deal with the hard input constraints. To support this algorithm, theoretical analyses of the closed-loop stability are given systematically.

This algorithm has the following three advantages: 1) high precision, 2) low online computational load, 3) maximized closed-loop stable region. Simulation results on a time-invariant Wiener-typed system with hard input constraints are performed, which validate the feasibility and superiority of this algorithm.

Also, this algorithm has somewhat limitations. A precondition is that some priori knowledge is available about the nonlinear block. Otherwise, Zeroin algorithm can not be applied. Consequently, in order to eliminate the limitation, our future research will focus on a more effective control algorithm to eliminate this requirement.

\section{ACKNOWLEDGMENT}

The authors would like to acknowledge the National Natural Science Foundation of China (NSFC) under Grant Nos. 60340420431and 60274020, and the Youth Founding Project of HUST.

\section{REFERENCES}

[1]A.D. Kalafatis, L Wang, Identification of Wiener-type nonlinear system in a noisy environment. INT.J.Control. 66(6),1997,pp:923-941

[2]A. Kalafatis, et al. A new approach to the identification of $\mathrm{pH}$ process based on the Wiener model, Chem.Eng.Science, vol.50, 1995,pp: 3693-3701.

[3]Chi-Tsong Chen, Linear System Theory and Design[B], 2000: 242-251

[4]E.F. George, M. A.Malcolm, C. B.Moler, Computer methods fro mathematical computations [B], Prentice-Hall, Inc., Englewood Cliffs, New Jersey, 1977,pp:156-166

[5]G.A. Pajunen, Identification of a $\mathrm{pH}$ process represented by a nonlinear Wiener model. IFAC Adaptive. Syst. Control Signal Processing, 1983,pp:91-95.

[6]Hassan K. Khalil , Nonlinear system[B], Prentice-Hall, Inc.,2002, 217-221

[7] H. Al-Duwaish, W. Naeem, Nonlinear model predictive control of Hammerstein and Wiener models using genetic algorithms, Control

Applications, 2001. (CCA '01). Proceedings of the 2001 IEEE International Conference on , Sept. 5th -7th ,2001 ,pp:465 - 469

[8] H. Chen. et al, Quasi-finite horizon nonlinear model predictive control scheme with guaranteed stability. Automatica, 34(10),1998,pp: 1205-1217 [9] H.H.J, Bloemen, et al. Wiener model identification and predictive control for dual composition control of a distillation column. Journal of Process Control, 11(6), 2001,pp: 601-620

[10]G.Z. Pang, Automatic theory[B], Press of University of Science and Technology of China, 1997

[11] J.C.Gómez, E.Baeyens, Identification of block-oriented nonlinear systems using orthonormal bases, Journal of Process Control, 14, 2004, p: 685-697

[12] J.J. Yang. Study on the model predictive control method of system with input constraints. Doctor dissertation of Northeast University of China. June, 2000.

[13] M. Szmaier, et al, Suboptimal control of linear systems with state and control inequality constrains. Proc. IEEE Conf. Dec. Contr.,

1997,pp:761-762

[14] O. Yamanaka, et al, Adaptive control and stability analysis of nonlinear systems using neural networks, Neural Networks,1997, International Conference on, 2424-2429

[15] S.J. Norquay, et al. Application of Wiener model predictive control (WMPC) to an industrial C2-splitter, Journal of Process Control, 9(6), 1999,pp: 461-473

[16]W. Greblicki, Nonparametric identification of Wiener systems by orthogonal series, IEEE Trans. Automatic Control, 39(10), 1994, pp: 2077-2086

[17]W. Greblicki, Nonparametric identification of Wiener systems, IEEE Trans. Automatic Control, 38(5), 1992, pp: 1487-1493

[18] X.J. Wang, et al, Weighting adaptive control of Wiener model based on multilayer feedforward neural networks, Proceeding of the 4th Word Congress on Intelligent Control and Automation. June 10th-14th, 2002, Shanghai, China 